\title{
Self-Configuring Active Services for Programmable Networks
}

\author{
Ralph Keller and Bernhard Plattner \\ Computer Engineering and Networks Laboratory \\ Swiss Federal Institute of Technology, Switzerland \\ \{keller | plattner\}atik.ee.ethz.ch
}

\begin{abstract}
This paper presents a service framework that allows routers to be programmed with user-specific code and coordinated such that the underlying network provides anticipated network services on behalf of applications. We describe how network services can be composed based on a high-level specification defining required and conditional processing steps, how capabilities about processing resources can be disseminated throughout the network by extending the OSPF protocol daemon, how services requirements are mapped onto the underlying network while minimizing both link and processing costs, and how service code is installed on selected nodes and forwarding state established along explicitly routed paths.
\end{abstract}

Keywords: Active networks, service specification, resource discovery, selfconfiguration, service deployment.

\section{Introduction}

During the last decades the original Internet architecture evolved dramatically with new functionality being added to the network layer to support a wide range of emerging applications. Network services such as firewalls, congestion control, media gateways, and traffic engineering all require a network that not only forwards packets based on the destination address, but also performs packet processing on nodes interior to the network. As a reaction to such application-specific packet handling requirements, router manufacturers have started to embed programmable elements into routers with the objective of providing network services in a more flexible way. However, deploying new services is usually a manual and time consuming process, requiring the installation of code on multiple routers distributed all over the network. Given the complexity of how services can be composed, the only feasible approach is to automate this process.

In this work we present a services infrastructure that allows router resources to be programmed and coordinated such that the underlying network provides anticipated services on behalf of applications. We have implemented the active network control software (ANCS) that offers a generic service abstraction and automates the configuration of processing resources to form network services. Our system accepts processing demands from applications based on a high-level service description, 
discovers available processing capabilities within the network, maps processing requirements onto the network, and configures the appropriate underlying resources such that the network provisions the expected service. In the remainder of this paper, we focus on all the mechanisms involved in the establishment of network services.

\section{A Framework for Self-Configuring Services}

In the following, we discuss the design rationales of our envisioned service framework that enables applications to coordinate network resources for building network services.

\subsection{Overall Network View}

To enable flexible services within a programmable network, there is a need for an additional control layer built on top of raw processing capabilities that facilitates the deployment and configuration of user-supplied code. The ANCS can be seen as a distributed system that automates the configuration of network resources to form services that applications use. The system accepts processing demands from applications using a network programming interface (NPI), discovers available processing resources, maps those processing requirements onto network resources, and configures the appropriate state on network nodes. The fact that nodes can perform this mapping autonomously on behalf of applications is a distinct feature of ANCS's design.

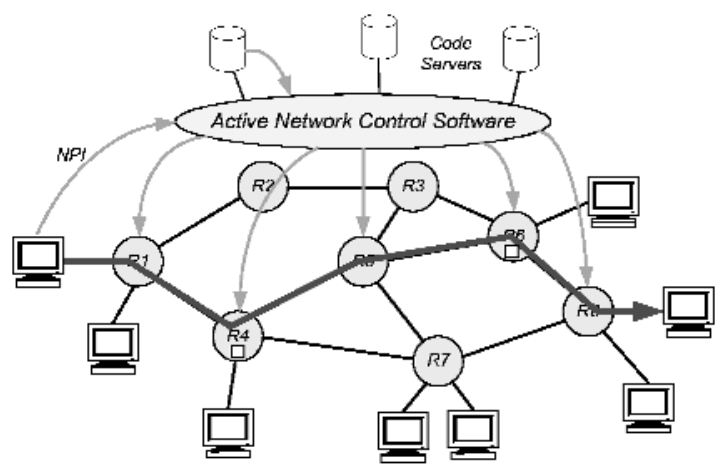

Fig. 1. Distributed network control software for active networks

Figure 1 illustrates our envisioned system where nodes participate in the establishment of application services. Each node continuously monitors the state of its locally available processing resources and distributes information it has on resources to its neighbors. Based on this scheme, each router learns about the location and capabilities of processing resources of other routers and propagates this information throughout the network. To set up network services, applications send a request (using the NPI) to the network control software running on the active nodes. 
The router that receives the request then tries to determine the network resources that are needed to satisfy the request. More specifically, the router computes the location for placing processing resources and communication channels connecting the peers with the processing nodes. If a feasible configuration could be computed, a signaling protocol then sets up the service by deploying code on the selected nodes and by establishing communication channels such that packets are routed through the processing functions. Packet processing code comes in form of router plugins [8] that are loaded into the execution environment of the data plane. If processing code has not been loaded previously, it is retrieved from a code server [3].

\subsection{Active Node Architecture}

Figure 2 illustrates the architecture of an individual active node, with the following components participating in the establishment of network services:

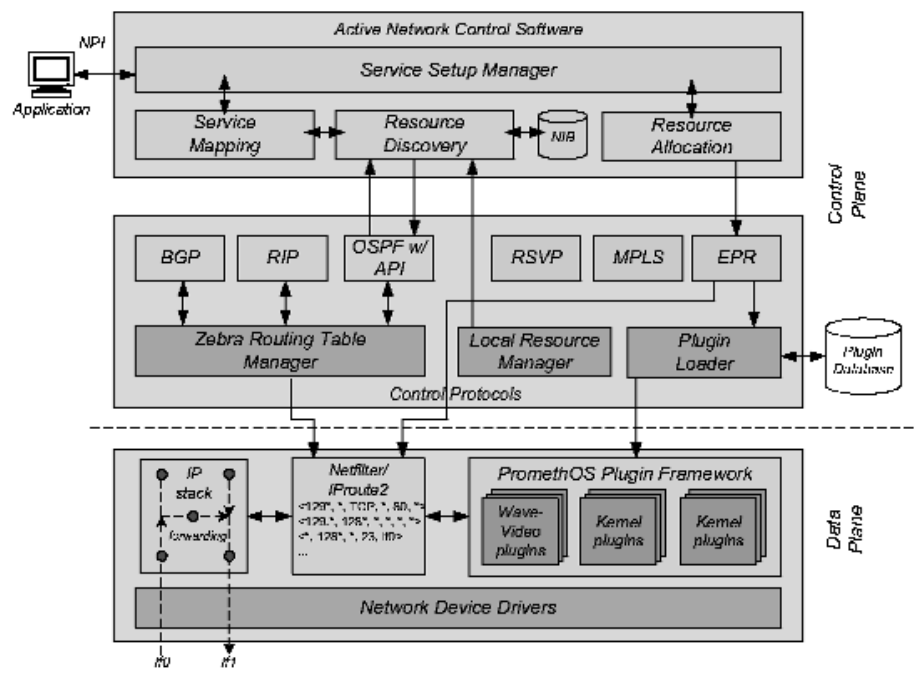

Fig. 2. Architecture of an individual active

- The active network control software accepts service establishment requests from applications, gathers information about processing resource capabilities, determines suitable locations for placing the packet processing functions, and initiates the configuration of associated network state.

- The OSPF protocol daemon has been extended with an API such that it can provide the ANCS with information about the topology and availability of processing resources.

- The explicit path routing (EPR) protocol installs processing code on selected nodes and establishes forwarding state such that traffic gets routed accordingly.

- The plugin loader retrieves router plugins from remote code servers when new packet processing code needs to be installed on a node. 
- The PromethOS plugin framework provides an environment for the dynamic loading of plugin classes, the creation of plugin instances as well as the execution of packet processing code.

In the following, we describe the ANCS in more detail.

\section{Active Network Control Software}

The ANCS can be seen as a new control layer implementing the functionality needed to set up services, thus freeing applications from knowing about the topology and details of low-level processing elements. Internally, the ANCS provides the following functions (see Figure 2 top):

- The service setup manager accepts service requests from applications using the NPI. To simplify the deployment of services, this interface needs to be designed in a way applications can abstract from the topology and low-level system details.

- A mapping mechanism translates high-level service requirements onto the network based on the constraints formulated by the application. This process determines the optimal location for placing processing functions and communication channels connecting the intermediate processing steps while guaranteeing application constraints.

- A resource discovery mechanism distributes information about the node's own resources and collects information about the processing capabilities from other active routers.

- A resource deployment mechanism sets up state across the network as computed by the mapping process. This includes the installation of processing code on selected routers and the establishment of appropriate forwarding state.

As depicted in Figure 2 the ANCS interacts with a number of underlying components present on the node. In particular, it monitors the state of its locally available processing resources from the local resource manager, and disseminates state information using the extended OSPF protocol to its neighbors. This way, each node learns about the topology and availability of resources embedded in the network. For the deployment of code and the establishment of network state across the network, the ANCS uses our proposed EPR signaling protocol. In the following, we describe each of the core functions involved in the establishment of services in more detail.

\subsection{Service Specification Using Active Pipes}

As a network programming interface between the user and the active network, we propose active pipes [7] that allow specification of communication and processing requirements by applications, regardless of the underlying topology and node-specific details. The idea of specifying transmission and processing requirements is to model a connection as a sequence of functions that have to be performed on the data stream. This concept is analogous to pipes in UNIX where data can be sent through a 
sequence of programs. In the context of active networks, these functions can be distributed on several nodes (unlike UNIX pipes were all processes execute on the same computer). Processing steps correspond to code modules that have to be instantiated along the path of the connection. However, an active pipe is a more general definition of the execution sequence since processing requirements can either be required or be conditional. That is, we distinguish between the following two types of code modules used in an active pipe:

- A required module provides a packet processing function that must be performed exactly once in the network. This functionality is essential for the correct operation of the application and cannot be omitted. Such a processing function typically changes the format of the data stream. An example is encryption, where the data stream must be encrypted exactly once within the network.

- A conditional module provides a functionality that is installed on all matching locations whenever a certain condition is satisfied. As a result the code module can be deployed multiple times along the path. If the condition is not satisfied at all, then no modules need to be installed. These code modules typically do not change the format of the data stream. A typical example is application-specific congestion control for video. If the data stream transits several congested links, congestion control modules will need to be installed before each of those links. If the data stream is not routed through any congested links, congestion control can be omitted.

Figure 3 depicts an active pipe for a scenario where a connection for sensitive data transmission should be established between two dislocated domains, and congestion control mechanisms desired on parts of the network where the stream is encrypted.

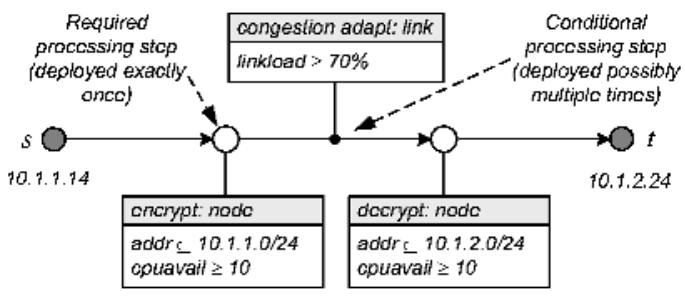

Fig. 3. Secure data transmission modeled using active pipe

The encryption and decryption steps are required exactly once within the source and destination domains, congestion control is optional (and deployed possibly multiple times) on links between the encryption and decryption steps. From a logical point of view, code modules can be deployed on either a node or along a link.

- Deployment on a node means that processing will be performed when the appropriate node is transited by the data traffic. An example for this type of processing is transcoding, where packets that go through the node need to be processed, regardless of the link from where the packet enters or leaves the node.

- Deployment on a link means that processing needs to happen when a given link is traversed. Here, the link that the data traffic enters or leaves a node is crucial for 
the application. For example for congestion control, processing needs to be performed when traffic is sent to congested links.

That is, for each processing step the application must specify whether processing should be performed when traffic either goes through a node or when traversing a link.

In addition to defining a sequence of required and conditional modules, the user can define individually for each processing step, installation conditions that describe the circumstances under which an active code module should be deployed. Since applications can have stringent requirements on the location of processing modules, each processing step can have multiple installation conditions to be considered for the deployment of a given function. Constraints restrict the location where processing can be performed. Each constraint is defined as a tuple <attr, rel, value $>$, expressing that attribute attr must fulfill value by the relation rel. If no constraints are defined for a processing step, all locations are considered to be valuable processing sites. In the example above, encryption and decryption must be placed within the given address ranges on nodes with sufficient processing cycles available, and congestion adaptation on the encrypted stream will be installed along all links where a certain congestion level is exceeded.

\subsection{Mapping Active Pipe onto Network}

Using active pipes, applications can now describe their processing requirements using a formalism that abstracts from the underlying physical network topology. However, this requires a mechanism that maps an active pipe onto the network, preferentially while minimizing network costs. In the following, we first explain how this mapping can be done in the case of a single required or conditional processing step, deployed on nodes or links. Then we look at how the mapping works for a combination of processing steps as in an active pipe. The main idea is to transform all the cases into specific shortest path problems and use the methods incrementally to generate a shortest path problem for the complete active pipe (see [4] and [7] for more details).

To state the mapping problems more formally, we use the following notation. We are given a directed graph, $G=(V, E)$, with a transmission cost $c(e)$, for each link $e \in E$, and a processing cost unit $c(v)$, for each node $v \in V$. Let the source be defined by $s$ and the destination by $t$. In graphs, we denote transmission costs on the links and the unit for processing costs within the node. For now we assume that all modules require a cost of 1 , that is, the cost for processing a module corresponds to $c(v)$. In addition, we assume that processing costs are scaled to match link cost metric. This convenience will allow us to express the mapping problem as a shortest-path problem.

\subsubsection{Required Processing}

For a single required processing step, we need to determine the location for placing the processing function and a path from the sources $s$ to the destination $t$ that transits the processing module (which can be placed only on one the candidate nodes). The 
mapping algorithm's objective is to minimize the sum of all transmission costs on links plus the processing cost for the module.
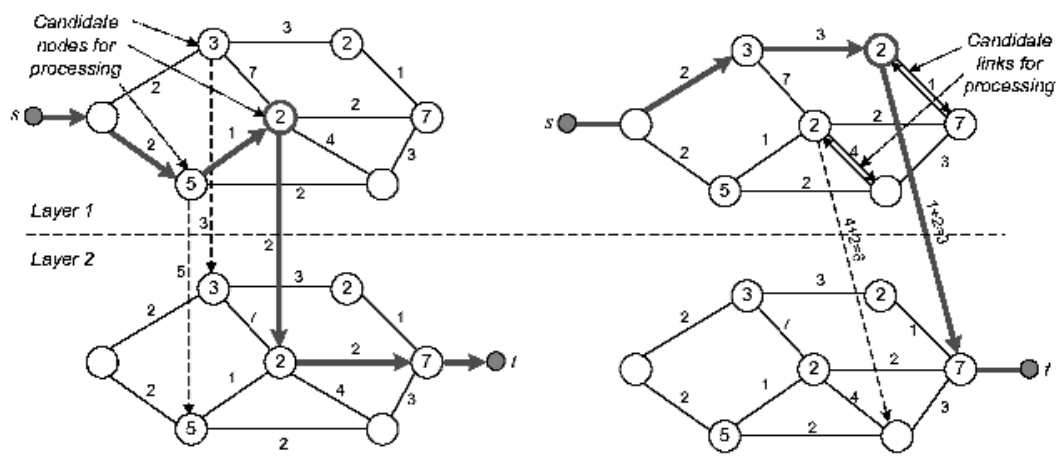

Fig. 4. Routing for required processing steps on a node (left) or on a link (right)

When processing should happen on a node (as for the encryption module), given the candidate set of nodes $N \subseteq V$ (defined by attribute constraints such as it must be placed within 10.1.1/24 and sufficient CPU cycles available), we need to determine the optimal node $n \in N$, where processing is performed. We can solve this problem by transforming it to a generic shortest path problem. As illustrated in Figure 4 (left), we modify the graph $G$ by making two copies which we identify as layer 1 and layer 2 . For each vertex $v$ in the initial graph, let $v_{1}$ denote the vertex in layer 1 of the target graph while $v_{2}$ denotes the vertex copy in layer 2. To model the processing of modules, we add edges between the two layers. For every node $n \in N$, where processing may occur, we add an edge $\left(n_{1}, n_{2}\right)$ in the target graph and let the link cost of $\left(n_{1}, n_{2}\right)$ be the processing cost on node $n, c(n)$. The source node in layer $1, s_{1}$, is the source for this new graph and the destination node in layer $2, t_{2}$, is the new destination node. This transformation ensures that the path from the source to the destination is forced to go through exactly one processing site. Now, to solve the routing problem with one mandatory processing site, we find a least-cost path in the target graph using a shortest path algorithm. The path can then be mapped back to the original graph by projecting the two layers onto a single layer and the processing is optimally performed where the path crosses the two layers.

In the case of processing on a link, the procedure of determining the optimal location is similar to the previous case, except for one small variation. Given the candidate set of links, $L \subseteq E$, the application wants the processing to be done on exactly one link among the set that needs to be traversed. Again, we transform the graph $G$ by making two copies as illustrated in Figure 4 (right). Now for every edge $e \in L$, which connects nodes $i$ and $j$, we add a new diagonal edge $\left(i_{1}, j_{2}\right)$ in the target graph between the two layers. The weight of this new edge is the sum of the processing cost at the node and the transmission cost of the link. This is given by the expression $c(k)+c(e)$, with $k=i$ when processing should be done at the node preceding the link (as assumed in the figure), or $k=j$ for processing at the succeeding node, respectively. The shortest path from $s_{1}$ to $t_{2}$ gives us an optimal path that transits 
one of the candidate links, with the node with the outgoing layer-crossing link denoting the optimal location for processing.

\subsubsection{Conditional Processing}

In the conditional processing case, active modules should be installed along the path at locations that satisfy a given condition. In the following, we look at the cases for conditional processing on nodes and links and describe how the locations for placing these modules can be determined.

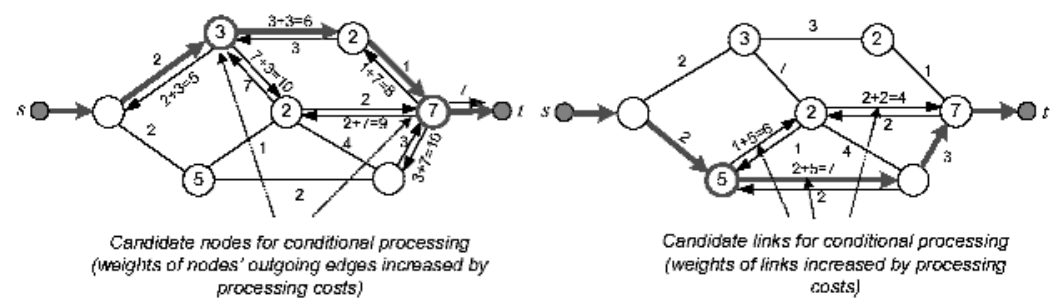

Fig. 5. Routing for conditional processing steps on nodes (left) and links (right)

In the case of conditional processing on nodes, we are given a candidate set of nodes, $N \subseteq V$, and we need to determine the set of nodes where the active module should be installed such that the path cost is optimal. More formally, suppose there exists a path $<s, v_{1}, v_{2} \ldots, t>$ from the source $s$ to the destination $t$, and if $v_{i} \in N$, then an active module should be installed on that node $v_{i}$. This can be solved as follows. For every node $v \in N$, let $\left\{e_{1}, e_{2}, e_{3}, \ldots e_{n}\right\}$ be the set of outgoing edges. As shown in Figure 5 (left), the graph transformation is to increase the edge weights of the outgoing links by the processing cost of the node, that is, $c_{\text {new }}\left(e_{i}\right)=c\left(e_{i}\right)+c(v)$, while source and destination remain the same.

In case of conditional processing on links, the application wants modules at all links in the path given a candidate set of links, $L \subseteq E$. An example for this scenario is congestion control, where congestion control modules need to be installed whenever a congested link is traversed. Similar to the conditional node case, all links belonging to the candidate set where processing could happen are marked with the processing step. The transformation on the graph is, as illustrated in Figure 5 (right), for every edge $e \in L$ to increase the edge weight by the processing cost of the node adjacent to that link. That is, $c_{\text {new }}(e)=c(e)+c(v)$, where $v$ is either the node preceding or succeeding the link $e$.

\subsubsection{Combination of Required and Conditional Processing}

The mapping for the processing steps in an active pipe includes a combination of required and conditional steps. Our approach is to combine the transformations described above and produce a target graph that represents the solution for the complete active pipe. 


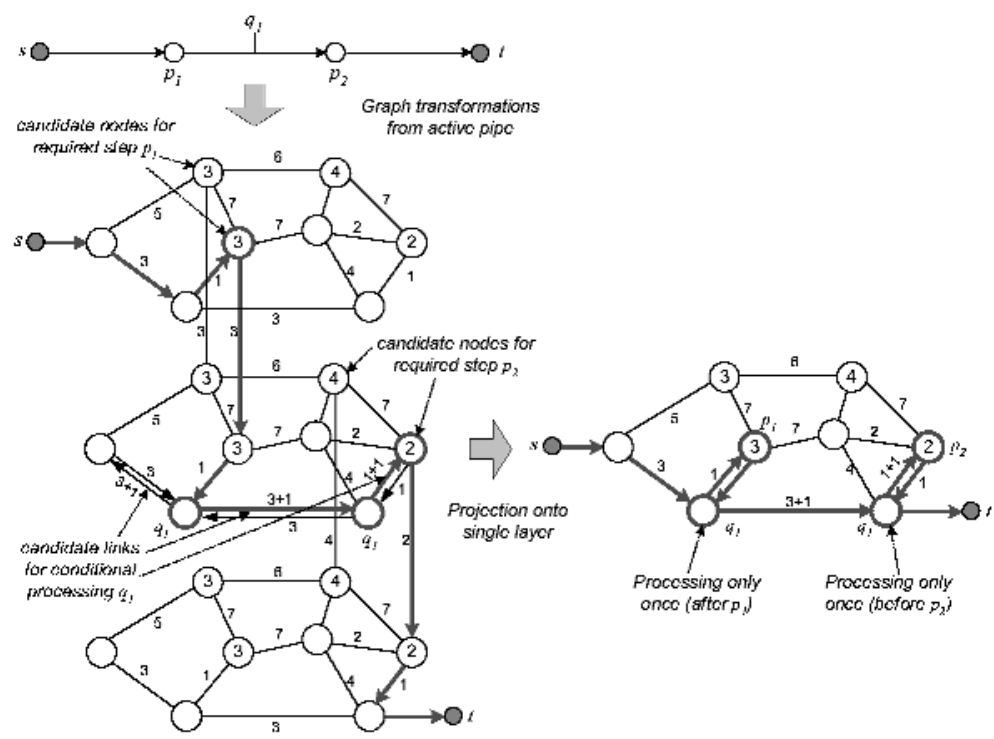

Fig. 6. Transformations for two required and one conditional processing steps

More formally, an active pipe includes of a source $s$, destination $t$, a set $P$ of required processing steps, and set $Q$ of conditional processing steps. For each required step $p_{i} \in P$ we extend the graph by adding a new layer according to the transformations defined previously. With $k$ the number of required processing steps, the target graph $G$ has $k+1$ layers, each layer representing a copy of the original graph. Between layers, we add edges in the target graph when a node is suitable for that processing step. Each conditional step $q_{j} \in Q$ results in modification of links weights of the current layer $c$. Initially, $c=1$, and whenever a new layer is being added, $c$ is incremented by 1 to reflect the most recently added layer. Each conditional processing step performs modifications of the current layer only. A number of conditional processing steps can be installed together on the same layer using a superposition of transformations, resulting in modifying link weights for each of the modules.

Figure 6 describes the graph transformations for the secure data transmission example presented in Figure 3. This active pipe contains the required encryption step $p_{1}$ and decryption step $p_{2}$, and a conditional processing step $q_{1}$ for congestion control on the encrypted stream. The target graph includes three layers. The candidate nodes suitable for a processing step on a given layer are indicated with arrows. The interlayer edges represent the required steps $p_{1}$ and $p_{2}$, and changes of the link weights on the middle layer represent conditional processing $q_{1}$. The resulting graph satisfies all processing requirements given by the active pipe because for each step a corresponding graph transformation has been performed. Solving the shortest path for the resulting graph returns the optimal place for $p_{1}, p_{2}$, and in addition all the locations for $q_{1}$.

Note that to compute a path for $k+1$ layers, the running time for a solution of the layered graph is essentially $k+1$ times the cost of computing a shortest path in the 
original graph, thus the total computational complexity remains polynomial and therefore scales to large networks. As shown in Figure 6, the solution can produce non-simple paths where a given node can be visited multiple times and a node can potentially be used more than once as a processing site. This fact must be taken into account by the signaling protocol.

\subsection{Resource Discovery Based on Extending OSPF}

Since the objective of our service framework is to facilitate the deployment of active services while hiding the topology and network-specific details from users, the service frameworks needs to be able to discover the underlying network topology needed for the mapping process. Thus, the framework requires a protocol that can distribute information about the (1) topology of the network and (2) the locations, capabilities, and availability of network-embedded processing resources.

First, to obtain information about the network topology, our approach is to directly access the link-state database of underlying routing protocols. Routing protocols such as OSPF John Moy, “OSPF Version 2," , IS-IS ISO DP 10589, “Intermediate System to Intermediate System Intra-Domain Routing Ex, and PNNI ATM Forum Technical Committee, "Private Network-Network Interface Specification Ve build an internal topological database describing the connectivity between routers. Each individual piece of this database represents a particular router's local state. Based on this linkstate database, we can compute a graph representing the network topology.

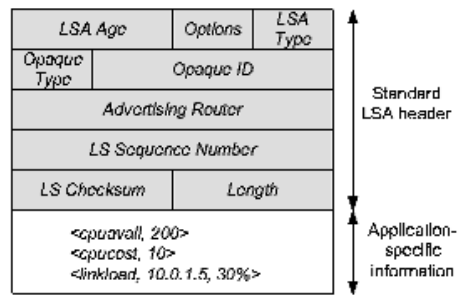

Fig. 7. Processing capabilities encoded as OSPF opaque LSAs

Second, to obtain information about processing resources embedded within the network we propose to use OSPF opaque link-state advertisements (LSA) [5]. Information within opaque LSAs is completely transparent for the routing protocol but can be interpreted by other instances such as the ANCS to identify processing resources.

An opaque LSA includes arbitrary information, typically encoded as a collection of type-length-value (TLV) objects, where each object carries information related to a particular network attribute (Figure 7). As shown in Figure 2, we have extended the OSPF protocol daemon with an API such that our ANCS can retrieve the link-state database as well as can originate opaque LSAs representing network attributes. The OSPF protocol then transparently distributes opaque LSAs to other routers. Since the link-state database built by the routing protocol provides complete connectivity information from which the topology graph including processing attributes can be generated quite easily. 


\subsection{Resource Allocation Protocol}

The final phase in deploying a network service is the installation and configuration of processing modules on selected nodes (as computed by the mapping algorithm) and the establishment of associated state across the network. In the context of active networks, conventional destination-based routing schemes are not sufficient since traffic needs to transit processing sites in a given order which is generally not the IP default path. Our proposed explicit path routing protocol [8] supports flow-based explicit path establishment for one-way, unicast flows routed through a predefined list of hops and the installation of plugin modules along such paths.

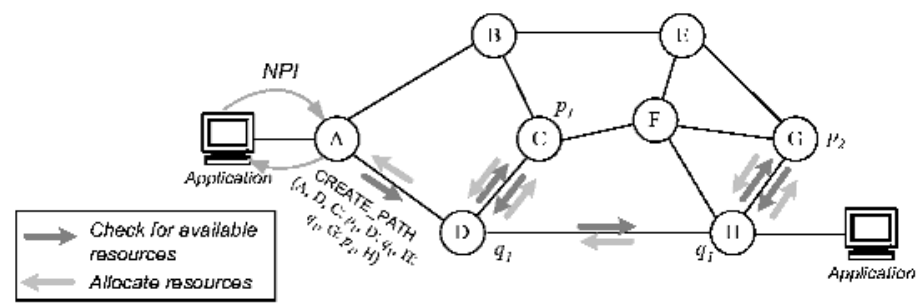

Fig. 8. Explicit Path Setup Protocol

Figure 8 illustrates the configuration of network state for the secure data example as computed by the mapping process (see Figure 6). The establishment of path state is based on two phases: In the first phase, the protocol verifies whether sufficient resources are available along the downstream path. Beginning at the source access router, each router checks whether the required resources are locally available and if true, reserves (but does not allocate yet) resources, and forwards the reservation request to the next node along the downstream path. This process is repeated until the destination access router is reached. Once the first phase of the setup process has been completed, it is assured that sufficient resources are available. In the second phase, the actual allocation of network resources takes place. This happens along the reverse path, that is, on all routers from the destination access router towards the source access router. This step includes the installation of flow-specific filters in the format <srcaddr/len, destaddr/len, sport, dport, proto, incoming interface> such that packets matching the filter are explicitly forwarded on the corresponding outgoing interface, and the installation and configuration of plugins and binding them to the filter. The incoming interface in the filter is used to discriminate when a non-simple path enters the same node several times. Once all state has been established along the path, the application is informed and can begin transmitting traffic.

If during the first phase a request is refused due to limited resources, the path setup process cannot continue and is aborted. The node then sends a reservation release message along the reverse path so that nodes that have already reserved resources can free them. When the establishment fails, the application is notified that the path could not be set up.

The EPR protocol uses TCP as the transport mechanism between EPR peers (hopby-hop) for sending control messages to establish paths, deploy plugins, and release resources. This guarantees reliable control messages distribution. However, for both path forwarding and plugin state stored on nodes, EPR uses soft-state to take into 
account that nodes and links are inherently unreliable and can fail. For that reason, an application that sets up a path is required to refresh the path (by sending the path establishment request periodically), otherwise nodes will purge path and plugin state once the time-out expires.

\section{Demonstration of Security Gateway Scenario}

We have implemented a fully functional prototype of our service framework, consisting of PromethOS [8] for loading and executing code, an extended OSPF daemon for resource discovery [9], the EPR [8] protocol for configuring network state, and the ANCS daemon to establish network services. All our code is free available [1].

To give the reader a feel for the simplicity of how services can be specified and deployed, we illustrate the commands necessary to configure a security gateway scenario.

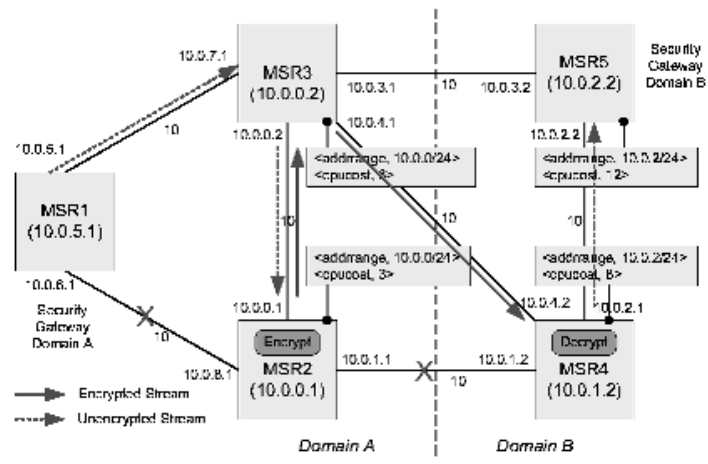

Fig. 9. Security gateway scenario

Figure 9 illustrates our test network. Nodes MSR1 and MSR5 are assumed to be security gateways that interconnect domain A and B and provide transparent encryption. We assume that the links MSR1 to MSR2 and MSR2 to MSR4 have failed and cannot be used. We require encryption to be placed on either MSR2 or MSR3 (MSR 1 does not offer processing), and decryption on MSR4. To simplify the scenario somewhat, the congestion control steps mentioned earlier have been omitted. We assume that node MSR2 has faster CPU capabilities and therefore set the processing costs on MSR 2 to 3 units while on MSR3 to 5 units, meaning that processing on MSR2 is favored if possible. Now to set up this scenario, we configure appropriate network state using the following statements (the CRYPT router plugin performing AES [11] encryption and decryption is assumed to have a cost of 10 processing units):

\# acm create --src 10.1.0.0/16 --dest 10.2.0.0/16 --from 10.0.5.1 --to 10.0.2.2 --plugin required node CRYPT E/mykey 10 --constraint addrrange subset 10.0.0/24

--constraint cpucost It 10

--plugin required node CRYPT D/mykey 10 --constraint addrrange subset 10.0.2/24 --constraint cpucost It 10 
Both processing steps are mandatory on nodes, but they have distinct installation conditions. The established service path for this scenario then looks as follows:

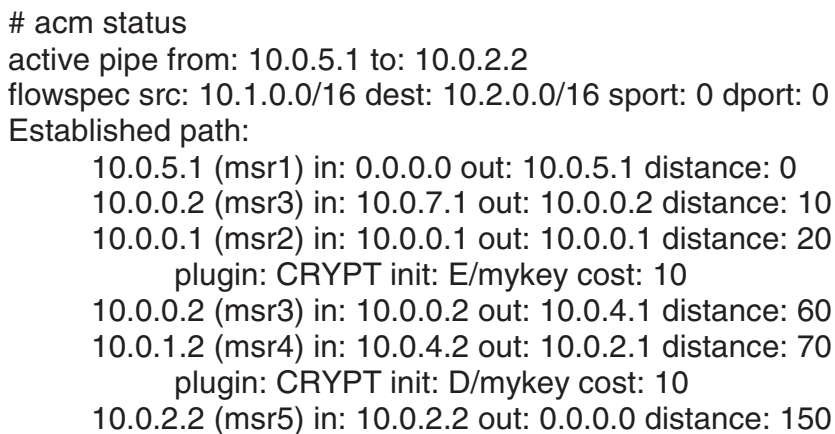

It is visible from the computation that the selected path is non-simple since MSR3 is being visited twice. Because processing on MSR2 is less expensive than on MSR3, the selected path is still better even when considering the additional link costs of the detour (twice the link cost between MSR2 and MSR3).

\section{Conclusions}

In this paper we have presented our active network control software that offers a generic service abstraction and automates the configuration of processing resources. Our system uses active pipes as a high-level programming abstraction to the active network, freeing applications from knowing about the underlying topology, location, and properties of processing nodes. Our system automatically discovers available processing capabilities within the network, maps processing demands onto available network resources based on an algorithm with polynomial complexity, and configures the network nodes such that traffic gets routed appropriately, thus significantly simplifies the use of active networks on behalf of applications.

\section{References}

[1] Active Network Control Software, Project Website, http://www.tik.ee.ethz.ch/ keller/ancs/

[2] ATM Forum Technical Committee, "Private Network-Network Interface Specification Version 1.0," March 1996.

[3] Sumi Choi, "Plugin Management," Washington University in St. Louis, Technical Report WUCS-00-04.

[4] Sumi Choi, Jonathan Turner, Tilman Wolf, "Configuring Sessions in Programmable Networks," In Proceedings of INFOCOM 2001, March 2001.

[5] Rob Coltun, "The OSPF Opaque LSA Option," RFC 2370, July 1998.

[6] ISO DP 10589, "Intermediate System to Intermediate System Intra-Domain Routing Exchange Protocol for use in Conjunction with the Protocol for Providing the Connectionless-mode Network Service (ISO 8473)," February 1990. 
[7] Ralph Keller, Jeyashankher Ramamirtham, Tilman Wolf, Bernhard Plattner, "Active Pipes: Service Composition for Programmable Networks", Milcom 2001, Lean VA, October 2001.

[8] Ralph Keller, Lukas Ruf, Amir Guindehi, Bernhard Plattner, "PromethOS: A Dynamically Extensible Router Architecture Supporting Explicit Routing," IWAN 2002, December 4-6, 2002 ETH Zürich, Switzerland.

[9] Ralph Keller, "Dissemination of Application-Specific Information using the OSPF Protocol”, Technical Report Nr. 181, TIK, ETH Zurich, November 2003.

[10] John Moy, "OSPF Version 2," RFC 2328, April 1998.

[11] National Institute of Standards and Technology (NIST), "Advanced Encryption Standard (AES)," FIPS PUBS 197, November 2001.

[12] Quagga/Zebra Project, Project Website, http://www.quagga.net/ 